\title{
Aqueductal Developmental Venous Anomaly Presenting with Mimic Symptoms of Idiopathic Normal Pressure Hydrocephalus in an Elderly Patient: A Case Report
}

\author{
Daisuke Kita, ${ }^{1}$ Cheho Park, ${ }^{1}$ and Yasuhiko Hayashi ${ }^{2}$
}

Developmental venous anomalies (DVAs) are generally asymptomatic; however, they can sometimes cause central nervous disorders. Aqueductal stenosis caused by DVAs is so rare that only 14 cases have been reported to date. Moreover, most patients are children or young adults, presenting with headaches or consciousness disturbances, associated with raised intracranial pressure. Here, we report on an 83-year-old man presenting with mimic symptoms of idiopathic normal pressure hydrocephalus (cognitive disorder, gait disturbance, and urinary urgency: Hakim's triad) because of obstructive hydrocephalus caused by a DVA located in the aqueduct. Endoscopic third ventriculostomy (ETV) was performed to relieve his symptoms, and the opening pressure of the lateral ventricle was recorded to be $10 \mathrm{~cm}-\mathrm{H}_{2} \mathrm{O}$. Endoscopic examination of the intraventricular system clearly revealed a vein within the aqueduct converging with the adjacent subependymal vessels. These findings were compatible with the characteristics of DVAs. His symptoms improved after the ETV. This case suggested that DVAs within the aqueduct, despite of their congenital nature, could give rise to decompensated obstructive hydrocephalus even in elderly patients, resulting in Hakim's triad.

Keywords: developmental venous anomaly, aqueductal stenosis, obstructive hydrocephalus, endoscopic third ventriculostomy

\section{Introduction}

Developmental venous anomalies (DVAs), previously termed venous malformations, are currently considered congenital nonpathologic anatomical variants of normal deep parenchymal veins, which are composed of mature venous vessels that lack malformed or neoplastic elements. ${ }^{1,2)}$ Although they are benign entities in nature, they occasionally cause headaches, seizures,

${ }^{1}$ Department of Neurosurgery, Noto General Hospital, Nanao, Ishikawa, Japan;

${ }^{2}$ Department of Neurosurgery, Kanazawa University Hospital, Kanazawa, Ishikawa, Japan

Received: October 12, 2018; Accepted: February 7, 2019

Online May 25, 2019

Copyright $\odot 2019$ by The Japan Neurosurgical Society

This work is licensed under a Creative Commons Attribution-

NonCommercial-NoDerivatives International License. and some focal neurological deficits. ${ }^{3,4)}$ Obstructive hydrocephalus as a complication of DVAs is so rare that only 14 cases of aqueductal stenosis (AS) have been reported so far. ${ }^{5,6}$ The previously reported cases of AS induced by DVAs were mainly in children and young adults, manifesting as chronic or intermittent headaches or consciousness disturbances resulting from elevated intracranial pressure (ICP). ${ }^{5,7)}$

Here, we present a case of AS caused by a DVA in an 83 -year-old man, manifesting symptoms of Hakim's triad, ${ }^{8)}$ with a normal ICP; this is the oldest patient reported upon so far. We present the radiological and endoscopic findings related to this uncommon entity, and then discuss the characteristics of clinical manifestations.

\section{Case Presentation}

An 83-year-old man, with Hakim's triad, cognitive disorder, gait disturbance, and urinary urgency over the past 4 months, was referred to our hospital. His general condition was good, with neither nausea nor headaches.

Magnetic resonance imaging (MRI) assessment showed supratentorial ventricular dilatation with ballooning of the third ventricle floor, which was consistent with radiological findings observed in obstructive hydrocephalus caused by AS (Figs. 1A-1C). A faint gadolinium-enhanced lesion was found at the dorsal part of the aqueduct, although it was difficult to determine whether the lesion contributed to the hydrocephalus (Fig. 1B, magnified image).

To resolve the symptoms associated with hydrocephalus, as well as to investigate the causative lesion, endoscopic third ventriculostomy (ETV) to inspect the ventricular system was carried out using a flexible neuro-fiber endoscope (VEF-V, Olympus Corporation, Tokyo, Japan). The right-side anterior horn of the lateral ventricle was punctured with least possible leakage of cerebrospinal fluid (CSF), and an initial opening pressure of $10 \mathrm{~cm}-\mathrm{H}_{2} \mathrm{O}$ was recorded. During endoscopy, we identified a vein accompanying the subependymal veins within the aqueduct (Fig. 1D). The appearance of this vascular lesion was compatible with the characteristics of a DVA, with an enlarged transcortical or subependymal collector vein draining radially arranged medullary veins. ETV was then performed in the conventional manner; his postoperative course was uneventful. His clinical symptoms remained good for 6 months after the operation, as summarized in Table 1. Follow-up MRI showed the flow void sign at the third ventricle floor, revealing patency of the stoma (Fig. 1E). 

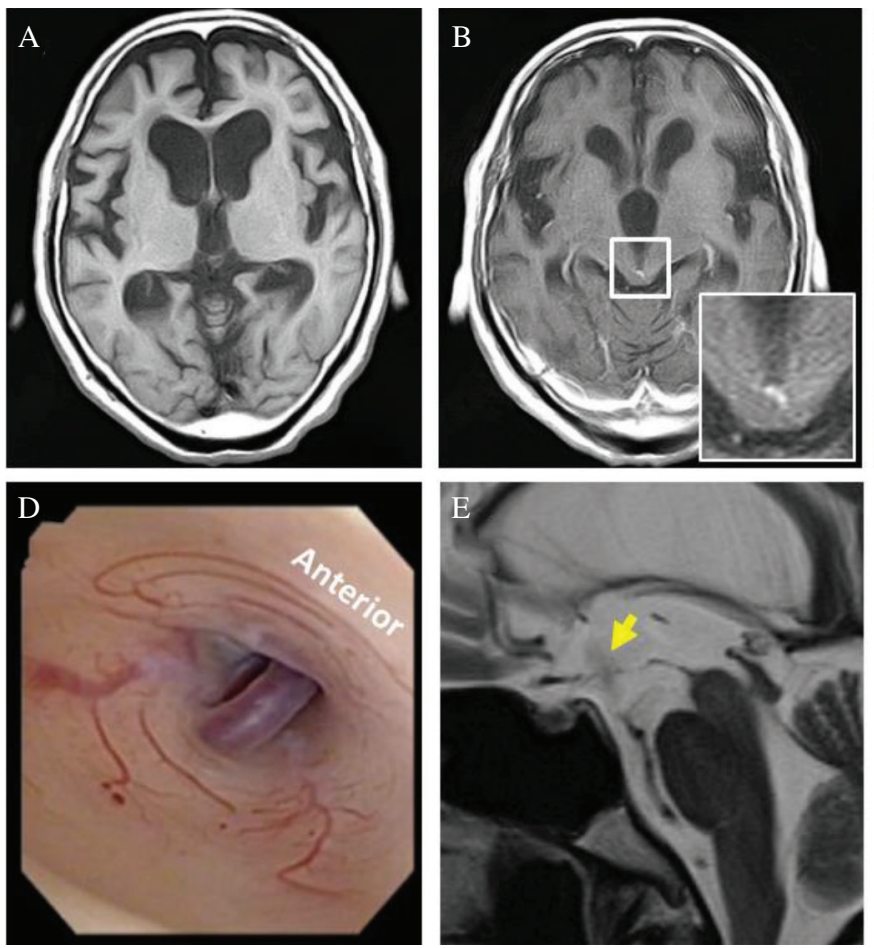

Table 1 Clinical evaluation in pre- and post-ETV

\begin{tabular}{lcc}
\hline Evaluations & Pre-ETV & Post-ETV \\
\hline MMSE (full score; 30) & 15 & 20 \\
3 m TUG (s) & 25 & 14 \\
INPHGS total score & 6 & 3 \\
$\quad$ Gait & 2 & 0 \\
Cognition & 3 & 2 \\
$\quad$ Incontinence & 1 & 0 \\
Modified Rankin Scale & 2 & 1 \\
\hline
\end{tabular}

ETV: endoscopic third ventriculostomy, INPHGS: idiopathic normal pressure hydrocephalus grading scale, TUG: timed up and go.

\section{Discussion}

Aqueductal stenosis may be induced by various central nervous system disorders, such as infection, hemorrhage, head trauma, tumors, cysts, or vascular lesions. ${ }^{910)}$ AS caused by DVAs is so rare that only 14 cases have been reported previously, all of which involved infants and relatively young individuals (0-58 years) (Table 2). ${ }^{5-7,11-20)}$ Apart from mental deterioration in the case of a 58-year-old woman ${ }^{11)}$ and seizure in the case of a 43 -year-old woman, ${ }^{14)}$ the main symptoms in the remaining 12 cases ( $0-42$ years) were severe or intermittent headaches and consciousness disturbances, which were closely associated with raised ICP.

Taking into consideration the congenital nature of DVAs, this case is quite unique, in that the intra-aqueductal DVA had been asymptomatic until he manifested with Hakim's triad at the age of 83. Although the underlying mechanisms of very late onset obstructive hydrocephalus in this patient is

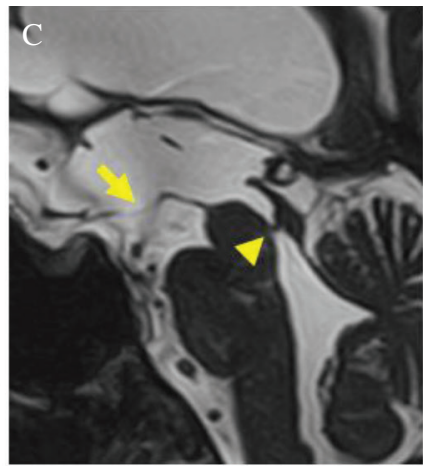

Fig. 1 (A) Axial image of $T_{1}$ MRI revealed enlargement of the lateral ventricles with an Evans index of 0.39. (B) Axial image of gadoliniumenhanced $T_{1}$ MRI revealed enlargement of the third ventricle (maximum diameter of $18.0 \mathrm{~mm}$ ) and a faint enhancement at the posterior part of the aqueduct (magnified image). (C) Preoperative sagittal image of heavily $\mathrm{T}_{2}$-weighted MRI revealed aqueductal stenosis (arrow head) and ballooning of the third ventricle floor (arrow). (D) Intraoperative endoscopic view showed a vein within the aqueduct and adjacent accumulation of subependymal vessels. (E) Postoperative sagittal image of $\mathrm{T}_{2}$-weighted MRI showed the flow artifact (arrow), revealing the CSF flow through the stoma made by endoscopic third ventriculostomy.

unclear, the clinical characteristics in this case are consistent with a previous study on idiopathic AS. Younger patients tend to have headaches with raised ICP, whereas older patients tend to show partial or complete Hakim's triad with normal ICP. ${ }^{21)}$ Long-standing intra-aquedactal DVAs, together with senile changes in the craniospinal environment, such as decrease in brain compliance, might induce decompensated obstructive hydrocephalus. ${ }^{22,23)}$

In cases of obstructive hydrocephalus caused by AS, ventriculoperitoneal shunting (VPS) with programmable pressure valves is another treatment choice. On the other hand, it was reported that the failure rate of initial VPS was much higher than ETV for adult patients with AS. ${ }^{24)}$ Regarding the first-line treatment option for patients with adult AS whose CSF absorbable capacity is expected to be preserved, ETV may be recommended rather than VPS, because ETV can prevent various problems related to VPS, such as overdrainage, shunt malfunction, or infection.

Moreover, in this case, use of a flexible endoscope for ETV enabled us to identify the DVA within the aqueduct, which was difficult to detect on preoperative MRI. Together with the previous eight cases involving the use of endoscopes (Table 2), ${ }^{5,14,17,19,20)}$ this case suggests that neuro-fiberscopes are not only useful for the treatment of AS but are also helpful in diagnostic exploration for AS with uncommon etiologies.

\section{Conclusion}

We presented a rare case of an elderly man with mimic symptoms of idiopathic normal pressure hydrocephalus (iNPH) caused by a DVA within the aqueduct. This case was quite unique for the following reasons: (1) the patient was much older than those in previous reports, (2) he showed 


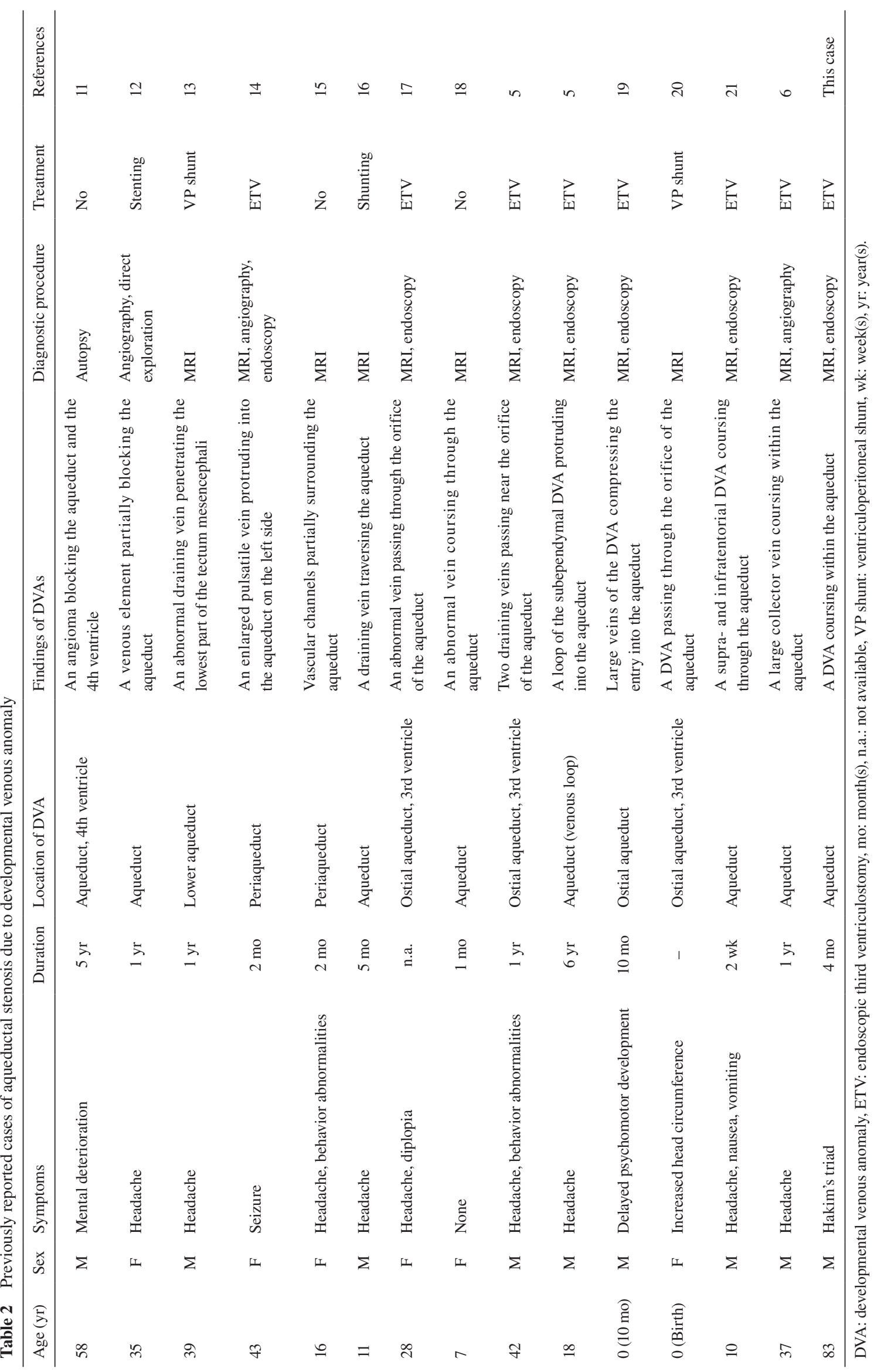


symptoms of Hakim's triad, which were resolved by ETV, and (3) an intra-aqueductal DVA was clearly shown on endoscopy. Even in elderly patients manifesting Hakim's triad, not only iNPH, but also obstructive AS caused by congenital lesions, such as DVAs shown in this case, should be considered as differential diagnosis.

\section{Conflicts of Interest Disclosure}

The authors have no conflict of interest to declare. This work was supported by a grant-in-aid for scientific research from Japanese Ministry of Health, Labor, and Welfare (\# H26-NANCHITOU-IPPAN-052, to D.K.).

\section{References}

1) Lasjaunias P, Burrows P, Planet C: Developmental venous anomalies (DVA): the so-called venous angioma. Neurosurg Rev 9: 233-242, 1986

2) Rammos SK, Maina R, Lanzino G: Developmental venous anomalies: current concepts and implications for management. Neurosurgery 65: 20-29; discussion 29-30, 2009

3) Naff NJ, Wemmer J, Hoenig-Rigamonti K, Rigamonti DR: A longitudinal study of patients with venous malformations: documentation of a negligible hemorrhage risk and benign natural history. Neurology 50: $1709-1714,1998$

4) Morioka T, Hashiguchi K, Nagata S, et al.: Epileptogenicity of supratentorial medullary venous malformation. Epilepsia 47: 365-370, 2006

5) Giannetti AV, Rodrigues RB, Trivelato FP: Venous lesions as a cause of sylvian aqueductal obstruction: case report. Neurosurgery 62: E1167-E1168; discussion E1168, 2008

6) Cavallo C, Faragò G, Broggi M, Ferroli P, Acerbi F: Developmental venous anomaly as a rare cause of obstructive hydrocephalus: literature review and a case report. J Neurosurg Sci: 06 Oct, 2015

7) Paulson D, Hwang SW, Whitehead WE, Curry DJ, Luerssen TG, Jea A: Aqueductal developmental venous anomaly as an unusual cause of congenital hydrocephalus: a case report and review of the literature. J Med Case Rep 6: 7, 2012

8) Adams RD, Fischer CM, Hakim S, Ojemann RG, Sweet WH: Symptomatic occult hydrocephalus with "normal" cerebrospinal-fluid pressure. A treatable syndrome. N Engl J Med 273: 117-126, 1965

9) Bennett RT, Allen PB, Miller JD: Non-tumoral stenosis of the aqueduct in adults. Surg Neurol 4: 523-527, 1975
10) Little JR, Houser OW, MacCarty CS: Clinical manifestations of aqueductal stenosis in adults. J Neurosurg 43: 546-552, 1975

11) Rosenheck C: Venous angioma of the sylvian aqueduct and the fourth ventricle associated with internal hydrocephalus and mental deterioration. Arch Neurol Psychiatry 38: 428-438, 1937

12) Avman N, Dinçer C: Venous malformation of the aqueduct of Sylvius treated by interventriculostomy: 15 years follow-up. Acta Neurochir (Wien) 52: 219-224, 1980

13) Watanabe A, Ishii R, Kamada M, Suzuki Y, Hirano K, Okamura H: Obstructive hydrocephalus caused by an abnormal vein in the aqueduct. Case report. J Neurosurg 75: 960-962, 1991

14) Oka K, Kumate S, Kibe M, Tomonaga M, Maehara F, Higashi Y: Aqueductal stenosis due to mesencephalic venous malformation: case report. Surg Neurol 40: 230-235, 1993

15) Blackmore CC, Mamourian AC: Aqueduct compression from venous angioma: MR findings. AJNR Am J Neuroradiol 17: 458460, 1996

16) Bannur U, Korah I, Chandy MJ: Midbrain venous angioma with obstructive hydrocephalus. Neurol India 50: 207-209, 2002

17) Sato S, Sonoda Y, Kuroki R, Kayama T: [A rare case of aqueductal stenosis due to venous angioma]. No To Shinkei 56: 1042-1046, 2004 (Japanese)

18) Yagmurlu B, Fitoz S, Atasoy C, Erden I, Deda G, Unal O: An unusual cause of hydrocephalus: aqueductal developmental venous anomaly. Eur Radiol 15: 1159-1162, 2005

19) Guhl S, Kirsch M, Lauffer H, Fritsch M, Schroeder HW: Unusual mesencephalic developmental venous anomaly causing obstructive hydrocephalus due to aqueductal stenosis. J Neurosurg Pediatr 8: 407-410, 2011

20) Inoue K, Yoshioka F, Nakahara Y, Kawashima M, Matsushima T: Obstructive hydrocephalus following aqueductal stenosis caused by supra- and infratentorial developmental venous anomaly: case report. Childs Nerv Syst 29: 329-334, 2013

21) Fukuhara T, Luciano MG: Clinical features of late-onset idiopathic aqueductal stenosis. Surg Neurol 55: 132-135; discussion 136-137, 2001

22) Czosnyka M, Czosnyka ZH, Whitfield PC, Donovan T, Pickard JD: Age dependence of cerebrospinal pressure-volume compensation in patients with hydrocephalus. J Neurosurg 94: 482-486, 2001

23) Czosnyka M, Czosnyka Z, Keong N, et al.: Pulse pressure waveform in hydrocephalus: what it is and what it isn't. Neurosurg Focus 22: E2, 2007

24) Oi S, Shimoda M, Shibata M, et al.: Pathophysiology of long-standing overt ventriculomegaly in adults. J Neurosurg 92: 933-940, 2000

Corresponding author:

Daisuke Kita, MD, PhD, Department of Neurosurgery, Yokohama Sakae Kyosai Hospital, 132 Katsura-cho, Sakae-ku, Yokohama, Kanagawa 247-8581, Japan.

$\triangle$ dk.md.phd@gmail.com 